\title{
Late-onset facial nerve degeneration after vestibular schwannoma surgery: incidence, putative mechanisms, and prevention
}

\author{
Prakash Sampath, M.D., Laurence D. Rhines, M.D., Michael J. Holliday, M.D., Henry Brem, M.D., \\ and Donlin M. Long, M.D., Ph.D.
}

Departments of Neurological Surgery and Otolaryngology-Head and Neck Surgery, Johns Hopkins Hospital, Baltimore, Maryland

Delayed facial nerve dysfunction after vestibular schwannoma surgery is a poorly understood phenomenon that has been reported to occur in 15 to $29 \%$ of patients undergoing microsurgery. It is a condition characterized by spontaneous deterioration of facial nerve function in a patient who has otherwise normal or near-normal facial function in the immediate postoperative period. This delayed paralysis is generally reported to occur in the first few days postsurgery, with the majority of patients eventually recovering their immediate postoperative facial function. However, infrequently, it can also occur more than 1 week after surgery (so-called late-onset facial nerve palsy).

The authors reviewed facial nerve outcome in 611 patients who underwent microsurgery between 1973 and 1994. The facial nerve was anatomically preserved in 596 patients $(97.5 \%)$, and $90 \%$ of patients had House-Brackmann[6] Grade 1 or 2 function 1 year after surgery. Late-onset facial dysfunction was seen in 13 patients $(2.1 \%)$. All of these had significant deterioration in facial nerve function between 1 and 4 weeks postoperatively, and all showed improvement by 1 year. In this study, the focus on these patients who developed late-onset facial palsy. The incidence, treatment strategies, and outcomes will be discussed with emphasis on possible pathophysiological mechanisms that contribute to this relatively rare condition.

Key Words * vestibular schwannoma * acoustic neuroma * facial nerve * delayed degeneration

In recent years, with advances in intraoperative cranial nerve monitoring and improvement in microsurgical techniques, considerable emphasis has been placed on preservation of facial nerve function during vestibular schwannoma surgery.[1,2,11,14-17,19] Given the medical, psychological, and functional sequelae of facial nerve weakness, $[13,23]$ close attention has been paid to factors that influence facial nerve outcome, and a number of authors have reported on their experience with these complex tumors. $[1,11,17,18,21]$ In recent years, the phenomenon of delayed facial nerve palsy has become evident.[10,12,22] This condition, characterized by spontaneous deterioration of facial nerve function in a patient who has otherwise normal or near-normal facial function in the immediate 
postoperative period, has been reported to occur in 15 to $29 \%$ of patients who underwent microsurgical resection of vestibular schwannomas. The natural history, prognosis, treatment, and pathophysiological mechanisms of delayed facial nerve dysfunction remain poorly understood. The majority of patients who develop this condition do so in the first few days after surgery. However, there is a small subset of patients who suffer deterioration of facial nerve function more than 1 week after surgery, and this condition has been termed late-onset facial nerve palsy.

To better understand late-onset facial nerve dysfunction we analyzed our experience with patients who underwent microsurgery at the Johns Hopkins Hospital between 1973 and 1994. We have recently reported on the long-term facial nerve outcome in 611 patients.[18] In this series, the facial nerve was anatomically preserved in 596 patients $(97.5 \%)$. Eighty-five percent of these patients had House-Brackmann Grade 1 or 2 function 6 months after surgery, and this percentage rose to $90 \%$ at 1 year postoperatively. Overall, we identified 13 patients $(2.1 \%)$ who developed late-onset facial nerve dysfunction. In this article, we focus on the treatment, outcome, and putative pathophysiological mechanisms in an effort to alert neurosurgeons and neurootologists to this rare phenomenon.

\section{CLINICAL MATERIAL AND METHODS}

An analysis of 611 patients who underwent microsurgical resection of vestibular schwannomas between September 1973 and January 1994 was undertaken. All patients who had preoperative facial nerve dysfunction, previous acoustic neuroma surgery or other treatment, unusual or atypical lesions, or poor follow-up results were excluded from the analysis. Patient charts, operative notes, neurocritical care-unit notes, and follow-up clinic notes were carefully reviewed. Of 611 patients, 13 were identified who developed late-onset facial nerve dysfunction, which was defined as profound deterioration in facial nerve function (House-Brackmann Grade 5 or 6) that occurred 1 week or more after surgery in patients with normal or nearly normal facial function in the immediate postoperative period. Nerve degeneration corresponded with fibrillation potentials on electromyographic studies until regeneration occurred. Facial function at 1 year after surgery was noted in each of these patients.

All patients underwent microsurgical resection of their vestibular schwannoma via a suboccipital or translabyrinthine approach. In patients with absent hearing and small-to-moderate-sized tumors, a translabyrinthine approach was used. In patients with preserved hearing or moderate to large tumor size, a retrosigmoid or suboccipital approach was used. Surgery was performed with continuous facial nerve monitoring in all but one patient. Meatal facial nerve decompression was performed in two patients undergoing translabyrinthine approaches at the time of original surgery, by using a previously described technique.[7] Of note, these two patients did not develop late-onset deterioration of facial nerve function.

\section{RESULTS}

Of 611 patients, 13 developed late-onset facial nerve paralysis, yielding an incidence of $2.1 \%$. As defined earlier, these patients had excellent facial nerve function in the early postoperative period (House-Brackmann Grade 1 or 2). Between 1 and 4 weeks after surgery, however, these patients had a profound deterioration in facial nerve function (House-Brackmann Grade 6) with nerve degeneration.

At 1-year follow up, 10 (77\%) of 13 patients had recovered to a House-Brackmann Grade 2; two (15.3\%) recovered to a Grade 3; and one patient (7.7\%) had Grade 4 function. Of note, all patients showed some facial nerve recovery following deterioration, but none regained normal facial nerve function (Grade 1). In addition, no patient required further surgery. 


\section{DISCUSSION}

Delayed facial nerve dysfunction after acoustic neuroma surgery has received relatively little attention in the literature. Its incidence in the early postoperative period has been reported to be as high as $29 \%,[10]$ and it has generally been regarded as a benign condition with excellent prognosis for full recovery.[10,12,22] Most of the reports describing delayed facial paralysis state that deterioration in facial nerve function occurs in the first few days after surgery.[10,12,22] However, the incidence of late-onset facial nerve dysfunction with nerve degeneration has been largely overlooked. This phenomenon, characterized by profound deterioration in facial function more than 1 week after surgery, may have a different natural history than delayed facial palsy that occurs earlier in the postoperative period, because the mechanisms of facial nerve injury may differ. In this paper, we elucidate this rare condition, report on its incidence and provide patient facial nerve outcomes 1 year after surgery. More important, we describe the putative pathophysiological mechanisms that may contribute to late-onset worsening of facial function and describe the preventive measures we take to minimize its occurrence. Our experience suggests that late-onset facial dysfunction may not have as good a prognosis as previously thought.

We find that the incidence of late-onset deterioration in facial function is quite low (2.1\%) and manifests in only a minority of patients. However, its occurrence can be quite alarming for the patient and almost always results in reevaluation by a physician, repeated imaging of the posterior fossa, and possibly rehospitalization. In our series, neuroimaging did not demonstrate unusual findings in any patient, such as a postoperative hematoma, to explain the mechanism of their facial nerve injury. We found invariably that patients had improved facial function by 1 year after surgery. This finding is consistent with those reported by other authors.[11,12,22] However, in our experience, no patient with late-onset facial paralysis with nerve degeneration regained perfect facial nerve function (House-Brackmann Grade 1). The majority of patients (10 of 13) still experienced some degree of synkinesis, giving them a House-Brackmann grade of 2; two patients had a grade of 3 and one patient a grade of 4, suggesting ongoing facial recovery may occur after 1 year.

The origin of late-onset facial nerve palsy after acoustic neuroma surgery is poorly understood. Consequently, preventing its occurrence remains a challenge. One possible mechanism has been suggested in which edematous entrapment of the facial nerve in the meatal foramen (the narrowest segment of the internal auditory canal[IAC]) may lead to nerve ischemia or necrosis. [8] When the translabyrinthine or middle fossa approach is used for tumor resection, decompression of the IAC from the meatal foramen to the geniculate ganglion may help to prevent delayed "entrapment" of the facial nerve.[5,7] Fisch[3] has described the meatal foramen as the narrowest segment of the IAC and suggested it was the site of greatest compression during Bell's palsy. This same pathophysiological mechanism may be used to explain why delayed facial nerve palsy is seen after acoustic surgery. This mechanism of facial nerve injury theoretically may occur late in the postoperative course, taking several days before the nerve is sufficiently swollen to become entrapped. However, clinical observations of delayed facial nerve paresis made by other authors, even after meatal decompression suggests,[4] alternative causative factors for delayed facial palsy.

Another possible mechanism may involve compromise of the blood supply to the facial nerve in the postoperative period. The facial nerve is supplied by three separate vascular systems.[9] The artery of labyrinth that supplies the intracranial portion of the nerve branches off the anterior inferior cerebellar artery and must be carefully preserved during suboccipital approaches to the cerebellopontine angle. In 
the IAC, the greater superficial petrosal branch of the middle meningeal artery supplies the geniculate ganglion and intracanalicular portion of the facial nerve. This vessel is identified and preserved early in the translabyrinthine approach to an acoustic tumor. Finally, the external carotid system gives rise to the stylomastoid artery that supplies the facial nerve as it exits the cranial vault near the stylomastoid foramen. The venous drainage of the facial nerve is variable and follows normal intracranial routes, but importantly, the veins and venules reside in the subarachnoid space surrounding the nerve. Devascularization of the facial nerve intraoperatively at the time of tumor dissection is unlikely to account for delayed facial nerve dysfunction, because one would suspect this would lead to immediate nerve injury. Arterial vasospasm from surgical manipulation, however, may lead to delayed nerve deterioration. This vasospasm may have an early muscular and a delayed inflammatory component similar to that seen after aneurysmal subarachnoid hemorrhage.[20] In addition, venous engorgement of the nerve with secondary stasis may lead to delayed swelling resulting in nerve injury.

Other putative mechanisms that may explain late-onset facial palsy with nerve degeneration include edema within the perineurium of the nerve, delayed immunological reactions, or simple traction (stretch) of the facial nerve in the cerebellopontine angle secondary to brain swelling or postoperative cerebrospinal fluid (CSF) leak. A viral origin, with reactivation of herpes zoster oticus, has also been implicated as a cause of delayed palsy.[4]

As a result of these theoretical mechanisms for late-onset facial nerve paralysis, we use several preventive measures in our patients. First, we administer perioperative steroid agents to minimize both cerebellar and perineurium swelling. Corticosteroids are known to diminish vasogenic edema in neural tissue, and we believe this potential benefit outweighs the minimal adverse effects of short-term steroid use. We also advocate postoperative head elevation, appropriate intravenous hydration, and maintenance of suitable electrolytes to reduce the likelihood of cerebral and neural swelling. Meticulous attention should also be given to bone waxing all mastoid air cells or using tissue glue in the porus acusticus, and ensuring tight dural closure to avoid postoperative CSF leak. If a CSF leak does occur, we routinely use serial lumbar puncture or lumbar drainage quite aggressively. Occasionally, judicious use of topical papaverine after tumor resection may aid in preventing vasospasm. Finally, although not appropriate for every patient, we believe meatal decompression should be considered when visible nerve discoloration or edema is present or when electromyographic responses to stimulation are poor.

In summary, late-onset facial paralysis with nerve degeneration after vestibular schwannoma surgery is a relatively rare but alarming occurrence. It may have several causative factors including neural entrapment, perineural edema, vasospasm, venous outflow disruption, traction of the nerve, and immunological phenomena, each of which may act alone or in concert. Several preventive measures may help to diminish the incidence of delayed facial nerve injury, and conservative treatment is usually indicated. In our experience, the vast majority of patients recover excellent facial nerve function (House-Brackmann Grade 2), but none regains completely normal facial function. Patients who develop this unusual condition should be counseled accordingly.

\section{References}

1. Cerullo LJ, Grutsch JF, Heiferman K, et al: The preservation of hearing and facial nerve function in a consecutive series of unilateral vestibular nerve schwannoma surgical patients (acoustic neuroma). Surg Neurol 39:485-493, 1993 
2. Ebersold MJ, Harner SG, Beatty CW, et al: Current results of the retrosigmoid approach to acoustic neurinoma. J Neurosurg 76:901-909, 1992

3. Fisch U: Surgery for Bell's palsy. Archo Otolaryngol 107:1-11, 1981

4. Gianoli GJ, Kartush JM: Delayed facial palsy after acoustic neuroma resection: the role of viral reaction. Am J Otol 17:625-659, 1996

5. Holliday MJ: Labyrinthine facial nerve decompression in acoustic neuroma surgery. Otol Head Neck Surg 93:48, 1985

6. House JW, Brackman DE: Facial nerve grading scale. Otolaryngol Head Neck Surg 93:146-147, 1985

7. Kartush JM, Graham MD, LaRouere MJ: Meatal decompression following acoustic neuroma resection: minimizing delayed facial palsy. Laryngoscope 96: 674-675, 1991

8. Kartush JM, Lundy LB: Facial nerve outcome in acoustic neuroma surgery. Otolaryngol Clinics North Am 25:623-647, 1992

9. Koos WT, Spetzler RF, Lang J: Color Atlas of Microsurgery, ed 2. Stuttgart: Thieme, 1993

10. Lalwani AK, Butt FY, Jackler RK, et al: Delayed onset facial nerve dysfunction following acoustic neuroma surgery. Am J Otol 16:758-764, 1995

11. Lalwani AK, Butt FY, Jackler RK, et al: Facial nerve outcome after acoustic neuroma surgery: a study from the era of cranial nerve monitoring. Otolaryngol Head Neck Surg 111:561-570, 1994

12. Megerian CA, McKenna MJ, Ojemann RG: Delayed facial paralysis after acoustic neuroma surgery: factors influencing recovery. Am J Otol 17:630-633, 1996

13. Parving A, Tos M, Thomsen J, et al: Some aspects of life quality after surgery for acoustic neuroma. Arch Otolaryngol Head Neck Surg 118:1061-1064, 1992

14. Post K, Eisenberg MB, Catalano PJ: Hearing preservation in vestibular schwannoma surgery: what factors influence outcome? J Neurosurg 83:191-196, 1995

15. Samii M, Matthies C: Management of 1000 vestibular schwannomas (acoustic neuromas): hearing function in 1000 tumor resections. Neurosurgery 40:248-262, 1997

16. Samii M, Matthies C: Management of 1000 vestibular schwannomas (acoustic neuromas): the facial nerve--preservation and restitution of function. Neurosurgery 40:684-695, 1997

17. Samii M, Matthies C: Management of 1000 vestibular schwannomas (acoustic neuromas): surgical management and results with an emphasis on complications and how to avoid them. Neurosurgery 40:11-23, 1997

18. Sampath P, Holliday MJ, Brem H, et al: Facial nerve injury in acoustic neuroma (vestibular schwanomma) surgery: etiology and preventon. J Neurosurg 87:60-66, 1997

19. Sampath P, Rhines LD, Goel R, et al: Cranial nerve preservation in contemporary vestibular schwanomma (acoustic neuroma) surgery. Crit Rev Neurosurg (In press, 1998) 
20. Sills AK Jr, Clatterbuck RE, Thompson RC, et al: Endothelial cell expression of intercellular adhesion molecule 1 in experimental posthemorrhagic vasospasm. Neurosurgery 41:453-461, 1997

21. Sterkers JM, Morrison GA, Sterkers O, et al: Preservation of facial, cochlear, and other nerve functions in acoustic neuroma treatment. Otolaryngol Head Neck Surg 110:145-155, 1994

22. Wedekind C, Hildebrandt G, Klug N: A case of delayed loss of facial nerve function after acoustic neuroma surgery. Zentralbl Neurochir 57:163-166, 1996

23. Wieland DA, Fickel V: Acoustic neuroma--the patient's perspective: subjective assessment of symptoms, diagnosis, therapy, and outcome in 541 patients. Laryngoscope 99:179-187, 1989

Manuscript received August 14, 1998.

Accepted in final form August 28, 1998.

Address reprint requests to: Donlin M. Long M.D., Ph.D., Department of Neurological Surgery, Meyer 7-109, The Johns Hopkins Hospital, Baltimore, Maryland, 21287-7709 